\title{
Radio Simulations of Upgoing Extensive Air Showers Observed from Low-Earth Orbit
}

\section{Andrés Romero-Wolf, ${ }^{a, *}$ in additional collaboration with Jaime Alvarez-Muñiz, ${ }^{b}$ Washington Carvalho, ${ }^{c}$ Harm Schoorlemmer ${ }^{d}$ and Enrique Zas $^{b}$ on behalf of the NuSpaceSim Collaboration}

(a complete list of authors can be found at the end of the proceedings)

${ }^{a}$ Jet Propulsion Laboratory, California Institute of Technology, Pasadena, CA 91109, USA

${ }^{b}$ Instituto Galego de Física de Alatas Enerxías IGFAE, Universidade de Santiago de Compostela, 15782 Santiago de Compostela, Spain

${ }^{c}$ Radboud Universiteit, Department of Astrophysics/IMAPP, Nijmegen, The Netherlands

${ }^{d}$ Max-Planck-Institute für Kernphysik, Heidelberg, Germany

E-mail: Andrew.Romero-Wolf@jpl.nasa.gov

Tau neutrinos interacting in the Earth can result in upgoing extensive air showers. These showers produce optical and radio emission that can be detected by orbital and suborbital platforms. We present results of radio emission simulations using ZHAireS for observation from low-Earth orbit as part of NASA's nuSpaceSim program to develop a comprehensive end-to-end simulation package to model these signals. Peculiar properties of the radio emission arise from the fact that these showers develop in extremely rarified portions of the Earth's atmosphere and, being observed from hundreds of kilometers distance, have distinct coherent emission features compared to ground observations.

$37^{\text {th }}$ International Cosmic Ray Conference (ICRC 2021)

July 12th - 23rd, 2021

Online - Berlin, Germany

\footnotetext{
*Presenter
} 


\section{Introduction}

Observing neutrinos above IceCube's most energetic event to date ( $\lesssim 10 \mathrm{PeV})[1]$ will require detectors with increasingly large exposures. At the highest energies, $\gtrsim 10^{19} \mathrm{eV}$, observation from the ground, although effective given the decade-scale lifetimes available, is ultimately limited by the large areas of detector arrays needed motivating suborbital [2-4] and orbital [5] observatories. NuSpaceSim [6] is a NASA-funded effort to estimate the sensitivity of optical and radio observatories in low-Earth orbit (LEO) to upgoing extensive air showers (EAS). These proceedings report on simulations of radio emission from upgoing EAS observed at LEO using the ZHAireS [7] software package.

\section{ZHAireS Simulations}

The ZHAireS simulation [7] package integrates the ZHS radio emission modeling in the frequency [8] and time domain [9] with the AIRES [10] extensive air shower simulations. This simulation package has been used for ground-based observatories, such as the Owens Valley Radio Observatory Long Wavelength Array [11] among other applications, and has been adapted to simulate upgoing air showers for the analysis of the ANITA air shower tau neutrino channel [12] at $\sim 37 \mathrm{~km}$ altitude and the BEACON high-elevation observatory concept [13] at 1-4 km altitudes. In this work, we apply the ZHAireS simulation package to provide predictions of the radio emission from upgoing extensive air showers for an observatory at the low-Earth orbit altitude of $525 \mathrm{~km}$ (Figure 1).

The setup for these simulations uses a single instance of a $10^{18} \mathrm{eV} \tau$-lepton electromagnetic decay $\left(\tau^{-} \rightarrow v_{\tau}+\bar{v}_{e}+e^{-}\right)$with $99 \%$ of the energy going to the electron. The decay was simulated with TAUOLA [14]. Following the approach of the ANITA simulations [12], the energy and momenta of each of the decay-product particles are injected into ZHAireS to initiate shower simulations with zenith angles of $55^{\circ}-85^{\circ}$ (in steps of $5^{\circ}$ ), $87^{\circ}$, and $89^{\circ}$, at altitudes of $0-9 \mathrm{~km}$ in steps of $1 \mathrm{~km}$. The electric field calculation in ZHAireS uses the ZHS algorithm in the time-domain [9] with $0.3 \mathrm{~ns}$ time bins. This temporal sampling corresponds to a bandwidth of $1.6 \mathrm{GHz}$. Examples of pulses produced by these simulations are shown in Figure 2. The electric field pulse peaks at the Cherenkov angle and is reduced in amplitude and bandwidth away from it. The view angle $\theta_{\text {view }}$ (Figure 1) sampling varies for each simulation geometry but is roughly $\sim 0.1^{\circ}$ steps ranging from $0^{\circ}-3^{\circ}$. In Figure 3 we show the beam pattern cuts of the geomagnetic radio emission for $50 \mathrm{MHz}$ sub-bands in the $50-1000 \mathrm{MHz}$ band. The general behavior is that the beam pattern is wider at low frequencies and narrows with decreasing peak amplitude at the Cherenkov angle with increasing frequency.

The geomagnetic field vector for each of these showers was set at a strength of $65 \mu \mathrm{T}$ in a direction orthogonal to the axis of propagation. When these results are implemented in a look-up table for simulations, the peak electric field strength scales as $\sin \alpha$, where $\alpha$ is the angle between the geomagnetic field. However, as will be shown in the next section, this relation breaks down for showers initiated at high altitudes where the atmosphere is significantly more rarified compared to showers that initiate closer to sea level. 


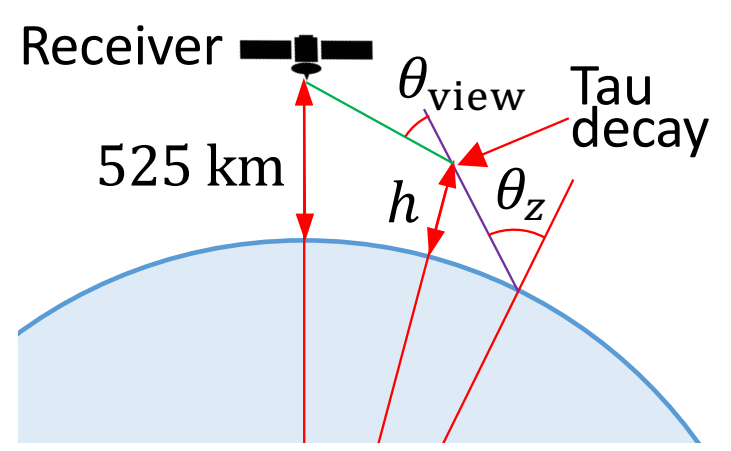

Figure 1: Simulation geometry. An upgoing tau exits the Earth at zenith angle $\theta_{z}$ and decays at an altitude $h$ above sea level. The radio emission is modeled for variety of view angles $\theta_{\text {view }}$ for a receiver at $525 \mathrm{~km}$ altitude.

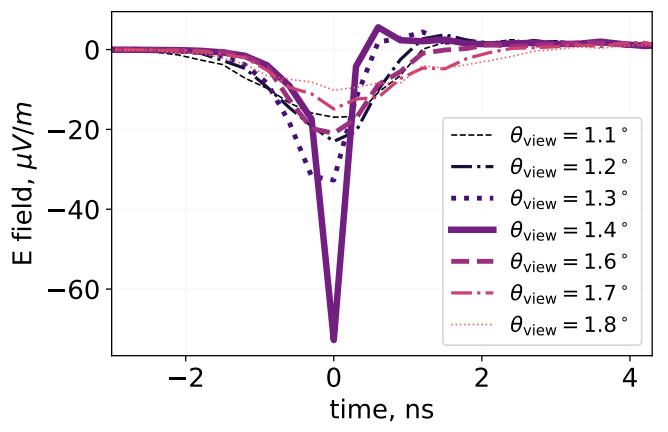

Figure 2: Example electric fields for an $87^{\circ}$ zenith angle upgoing extensive air shower from a tau decay at ground altitude observed at $525 \mathrm{~km}$. The Cherenkov angle is at $\sim 1.4^{\circ}$ for this geometry. The waveforms shown are unfiltered.

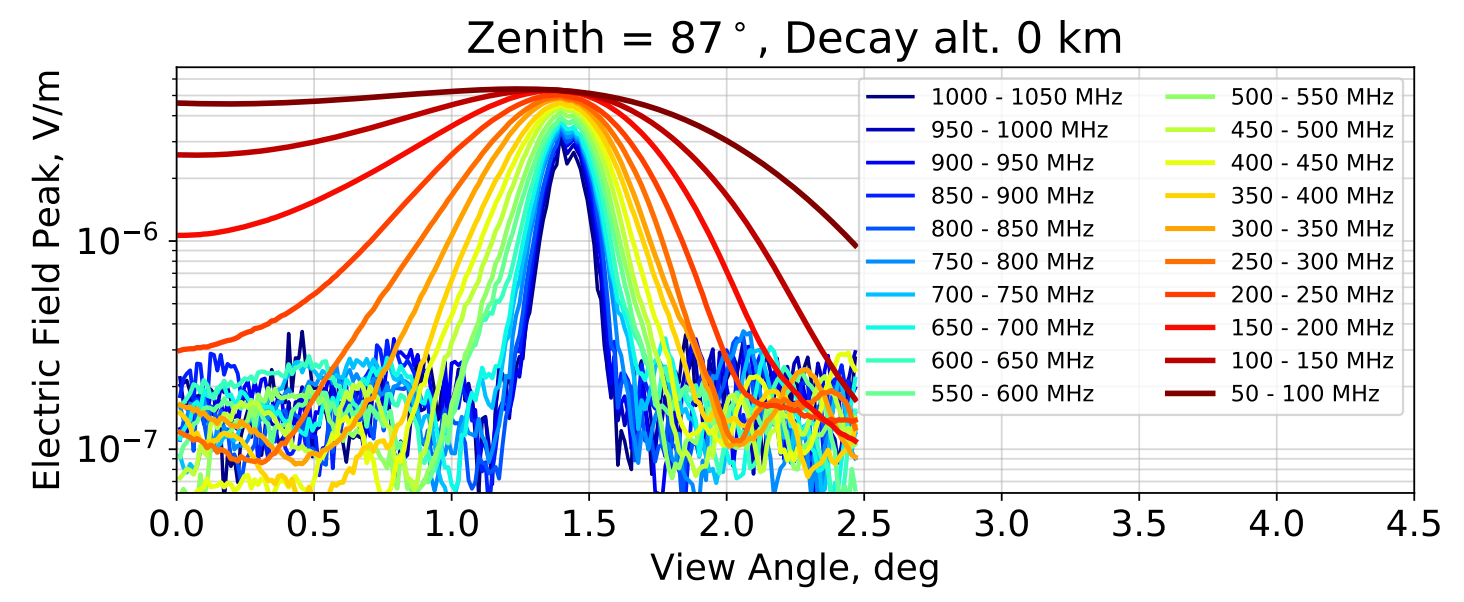

Figure 3: Example radio emission profiles for an $87^{\circ}$ zenith angle upgoing extensive air shower from a tau decay at ground altitude observed at $525 \mathrm{~km}$.

\section{Results}

\subsection{Shower Characteristics}

A subset of the results that will be implemented in an initial version of nuSpaceSim are shown in Figure 4. We have taken two bands: $30-300 \mathrm{MHz}$ also known as the very-high frequency (VHF) band and 300-1000 MHz, which is part of the ultra-high frequency (UHF) band. While we do have full flexibility to choose bands based on the ZHAireS outputs, we start with these delimitations because they correspond to bands used in radio-frequency engineering and each has a corresponding set of technological developments that would be applied to a future implementation.

In the UHF band, there is a clearly defined Cherenkov angle at $80^{\circ}$ zenith angles with the peak view angle shifting to lower values as the decay altitude increases. This effect is due to the 

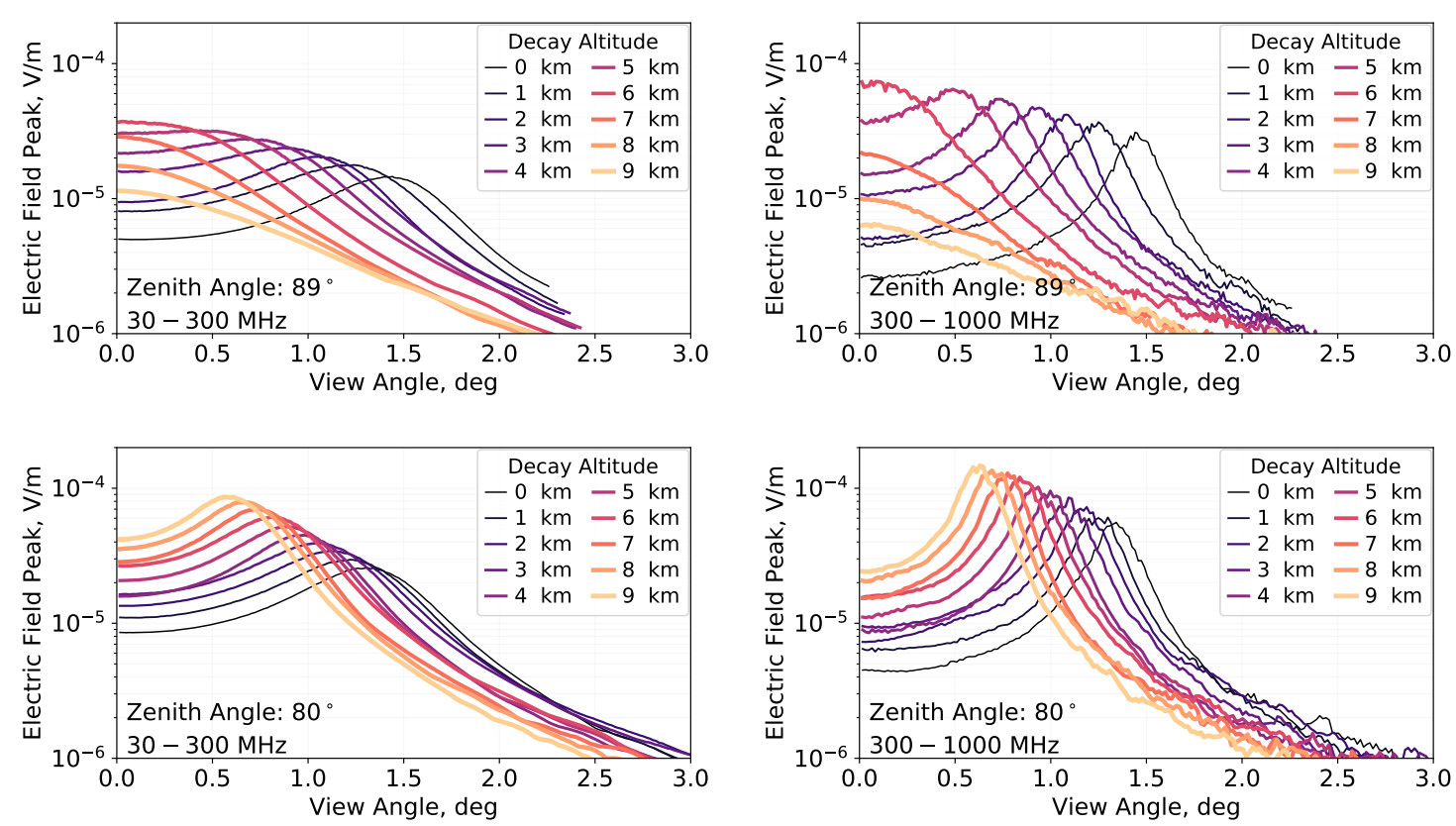

Figure 4: Radio emission profiles upgoing extensive air shower from a tau decay at ground altitude observed at $525 \mathrm{~km}$. Top left is for a shower zenith angle of $89^{\circ}$ in the $30-300 \mathrm{MHz}$ band while top right is for the same zenith angle in the $300-1000 \mathrm{MHz}$ band. The bottom left and right at for a shower zenith angle of $80^{\circ}$ in the 30-300 MHz band (bottom left) and 300-1000 MHz band (bottom right).

decreasing atmospheric density over the shower development with increasing decay altitude. At a zenith angle of $89^{\circ}$ the simulations show also indicate a trend of decreasing Cherenkov angle with decay altitude although it is more drastic than at $80^{\circ}$. This effect is unexpected since the shower should, in principle, evolve over a denser atmospheric profile at a zenith angle of $89^{\circ}$ than at $80^{\circ}$. The root cause of this difference, whether physical (which seems unlikely) or due to the limitations of the ZHAireS simulations, is currently under investigation.

\subsection{Saturation of Geomagnetic Radiation}

One of the novel features found in these simulations is the saturation of geomagnetic radio emission for high altitude upgoing air showers. In Figure 5 we show the dependence of the peak electric field emission as a function of geomagnetic field inclination angle $I_{B}$. As mentioned above, the simulations for the previous sections all had the geomagnetic field orthogonal to the shower axis of propagation (i.e. $I_{B}=\theta_{z}-90^{\circ}$ ). It is found that for showers initiated at high altitudes and steeply upgoing zenith angles, the geomagnetic field saturates above a value of $|B \sin \alpha|$ that varies for each shower zenith angle and decay altitude. This is a deviation from the conventional $B \sin \alpha$ dependence on the radio emission strength known from ground-based observations.

We suspect this effect is due to a balance between the geomagnetic deflection of the electrons and positrons in the shower and multiple scattering interaction length. The left panels of Figure 5 show that for a steeply upgoing shower $\left(\theta_{z}=50^{\circ}\right)$ the saturation effect becomes stronger for showers with increasing altitude. The right panels of Figure 5 show that for a shower with a more horizontal zenith angle $\left(\theta_{z}=87^{\circ}\right)$, the saturation effect is not noticeable and the emission follows the expected 

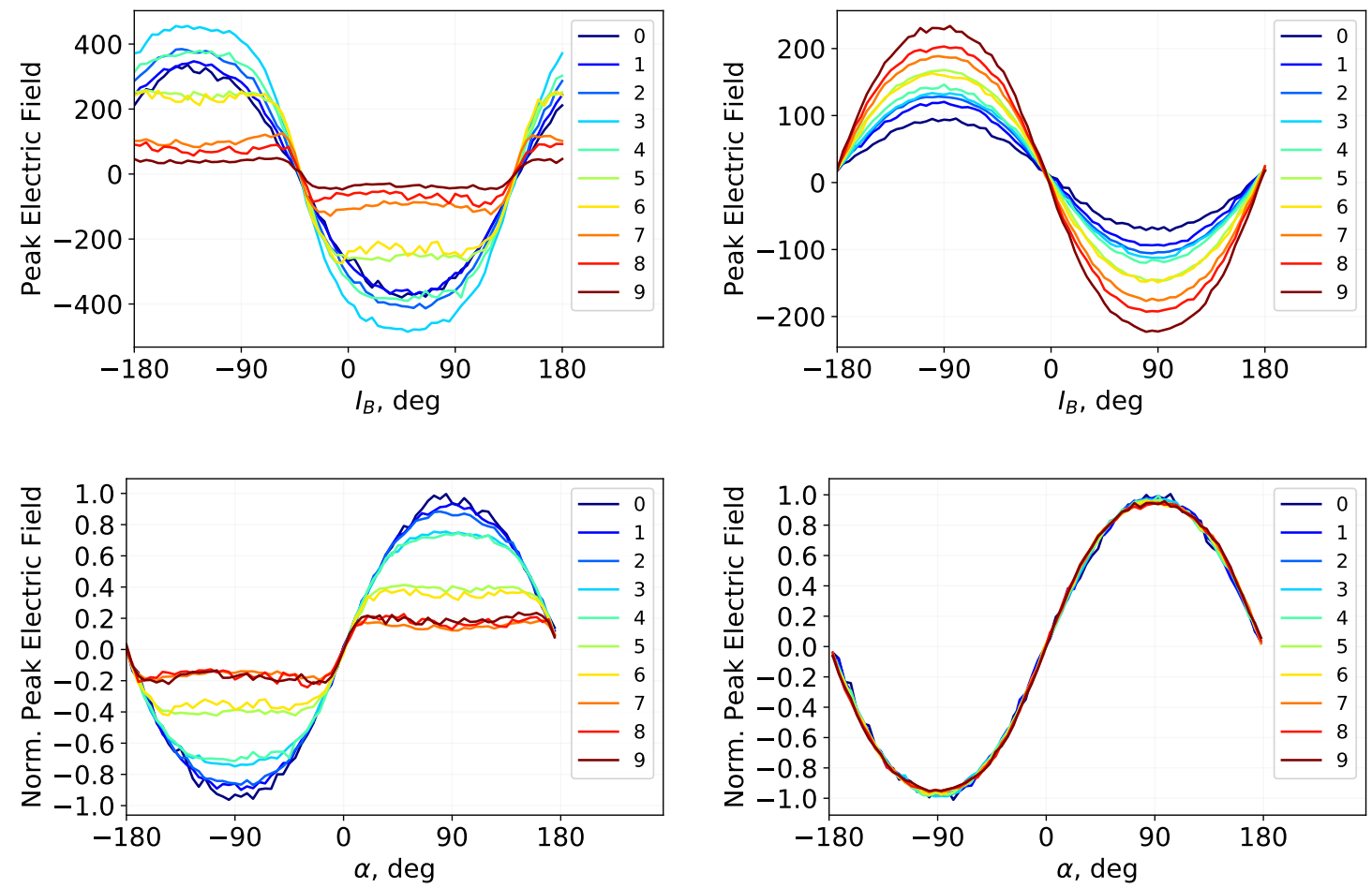

Figure 5: Geomagnetic field angle dependence on radio emission. Top left: the peak electric field value as a function of geomagnetic field inclination angle $I_{B}$ for a $50^{\circ}$ zenith angle shower. The colors indicate the decay altitude of each shower. Bottom left: the trends in the plot above have been fitted to a clipped sine wave and normalized by the peak amplitude of the fitted sinusoid and plotted in terms of the angle between the geomagnetic field vector and the shower axis $(\alpha)$ to illustrate the effect of the geomagnetic emission clipping fraction. Right top and bottom are the same as the left top and bottom, respectively, but for a shower zenith angle of $87^{\circ}$.

$B \sin \alpha$ dependence. The steeply upgoing shower will evolve into increasingly more rarified media in which the electrons and positrons can be deflected significantly away from the Cherenkov angle at the track level significantly suppressing the net radio emission.

\section{Discussion \& Outlook}

The results from these air showers are being used to produce look-up tables to be implemented for radio observatory simulations with nuSpaceSim. The radio emission is scaled with the shower energy, following the approach used for ANITA and BEACON, where it was shown that this approximation does not result in significant deviations [12].

We are finding that the behavior for high altitude showers at $89^{\circ}$ zenith angle is not producing high frequency emission while high-altitude showers at $80^{\circ}$ do contain high frequency emission. This is indicating a potential systematic error at high zenith angles which is possibly associated with the atmospheric index of refraction model implemented in ZHAireS. The results reported here, particularly at the highest zenith angles, should be taken as preliminary while this behavior is under investigation. 
The finding that the emission saturates for steeply upgoing showers at high decay altitudes will also be further investigated. The hypothesis that this is due to an increased multiple scattering interaction length of electrons and positrons in the shower compared to the geomagnetic deflection dominating can be tested in more detail using ZHAireS or simplified versions of the particle propagation simulations. This will have implications for the energy reconstruction of steeply upgoing air showers from future space-based observatories.

\section{Conclusions}

In these proceedings we have presented the first results of radio emission from detailed tracklevel upgoing air shower simulations as observed from low-Earth altitude. While the results share many similar features with regards to the radio emission beam pattern from similar simulations at lower altitudes for ANITA and BEACON, there is an as-of-yet unexplained behavior for nearly horizontal showers which is currently under investigation and these results should be taken as preliminary. The main new finding from this exercise is the saturation of geomagnetic radio emission for steep upgoing shower initiated at high altitude.

Acknowledgements: The research was carried out at the Jet Propulsion Laboratory, California Institute of Technology, under a contract with the National Aeronautics and Space Administration (80NM0018D0004). C2021 California Institute of Technology. All rights reserved. This work was sponsored under NASA grant 17-APRA17-0066. J. AM and E.Z. thank the following. This work has received financial support from Xunta de Galicia (Centro singular de investigación de Galicia accreditation 2019-2022), by European Union ERDF, by the "María de Maeztu" Units of Excellence program MDM-2016-0692, the Spanish Research State Agency and from Ministerio de Ciencia e Innovación PID2019-105544GB-I00 and RED2018-102661-T (RENATA).

\section{References}

[1] M. Aartsen et al. (IceCube Collaboration), "Characteristics of the Diffuse Astrophysical Electron and Tau Neutrino Flux with Six Years of IceCube High Energy Cascade Data", Phys. Rev. Lett. 125, 121104, (2020).

[2] P. W. Gorham et al. (ANITA Collaboration), "The Antarctic Impulsive Transient Antenna ultra-high energy neutrino detector: Design, performance, and sensitivity for the 2006-2007 balloon flight", Astroparticle Physics 32, 10, (2009).

[3] S. Bacholle et al. (JEM-EUSO Collaboration), "The EUSO-SPB instrument", 35th International Cosmic Ray Conference, PoS(ICRC2017)384, (2017).

[4] Q. Abarr et al. (PUEO Collaboration), "The Payload for Ultrahigh Energy Observations (PUEO): A White Paper", accepted for publication in the Journal of Instrumentation, arXiv:2010.02892 (2021).

[5] A. Olinto et al. (POEMMA Collaboration), "The POEMMA (Probe of Extreme MultiMessenger Astrophysics) observatory", Journal of Cosmology and Astroparticle Physics 2021, 007, (2021). 
[6] J. Krizmanic et al. (NuSPaceSim Collaboration), "nuSpaceSim: A Comprehensive Neutrino Simulation Package for Space-based \& Suborbital Experiments", 36th International Cosmic Ray Conference, PoS(ICRC2019)936, (2019).

[7] J. Alvarez-Muñiz et al., "Monte Carlo simulations of radio pulses in atmospheric showers using ZHAireS", Astroparticle Physics 35, 325, (2012).

[8] E. Zas et al., "Electromagnetic pulses from high-energy showers: Implications for neutrino detection", Phys. Rev. D 45, 362, (1992).

[9] J. Alvarez-Muñiz et al., "Čerenkov radio pulses from electromagnetic showers in the time domain", Phys. Rev. D 81, 123009, (2010).

[10] S. J. Sciutto, "AIRES: A system for air shower simulations", astro-ph/9911331, (1999).

[11] W. R. Carvalho et al., "Estimating the sensitivity of the expanded OVRO-LWA array to cosmicray primary composition", 36th International Cosmic Ray Conference, PoS(ICRC2019)211, (2019).

[12] A. Romero-Wolf et al. (ANITA Collaboration), "Comprehensive analysis of anomalous ANITA events disfavors a diffuse tau-neutrino flux origin", Phys. Rev. D 99, 063011, (2019).

[13] S. Wissel et al., "Prospects for high-elevation radio detection of $>100 \mathrm{PeV}$ tau neutrinos ", Journal of Cosmology and Astroparticle Physics 2020, 065, (2020).

[14] S. Jadach et al., "The tau decay library TAUOLA: Version 2.4", Comput. Phys. Commun. 76, 361 (1993). 


\section{+ NuSpaceSim $v$ SpaceSim Collaboration}

Yosui Akaike $^{1}$, Luis Anchordoqui ${ }^{2}$, Douglas Bergman ${ }^{3}$, Isaac Buckland ${ }^{3}$, Austin Cummings ${ }^{4}$, Johannes Eser ${ }^{5}$, Claire Guépin ${ }^{6}$, John F. Krizmanic $^{7,8,9}$, Simon Mackovjak ${ }^{10}$, Angela Olinto ${ }^{5}$, Thomas Paul ${ }^{2}$, Sameer Patel ${ }^{11}$, Alex Reustle ${ }^{9,12}$, Andrew Romero-Wolf ${ }^{13}$, Mary Hall Reno ${ }^{11}$, Fred Sarazin ${ }^{14}$, Tonia Venters ${ }^{9}$ Lawrence Wiencke $^{14}$, Stephanie Wissel ${ }^{4}$

${ }^{1}$ Waseda Institute for Science and Engineering, Waseda University, Shinjuku, Tokyo, Japan, ${ }^{2}$ Department of Physics and Astronomy, Lehman College, City University of New York, New York, New York, 10468 USA, ${ }^{3}$ Department of Physics and Astronomy, University of Utah, Salt Lake City, Utah 84112 USA, ${ }^{4}$ Department of Physics, Pennsylvania State University, State College, Pennsylvania 16801 USA, ${ }^{5}$ Department of Astronomy and Astrophysics University of Chicago, Chicago, Illinois 60637 USA, ${ }^{6}$ Department of Astronomy, University of Maryland, College Park, College Park, Maryland 20742 USA, ${ }^{7}$ Center for Space Sciences and Technology, University of Maryland, Baltimore County, Baltimore, Maryland 21250 USA, ${ }^{8}$ CRESST, ${ }^{9}$ NASA/Goddard Space Flight Center, Greenbelt, Maryland 20771 USA, ${ }^{10}$ Institute of Experimental Physics, Slovak Academy of Sciences, Kosice, Slovakia, ${ }^{11}$ Department of Physics and Astronomy, University of Iowa, Iowa City, Iowa 52242 USA, ${ }^{12}$ INNOVIM, ${ }^{13}$ Jet Propulsion Laboratory, California Institute of Technology, Pasadena, California 91109, USA, ${ }^{14}$ Department of Physics, Colorado School of Mines, Golden, Colorado 80401 USA 